\title{
Le concept de la nation chez Jean Froissart
}

\section{Věra Vejrychová}

\section{OpenEdition}

\section{Journals}

Édition électronique

URL : http://journals.openedition.org/ifha/8043

DOI : 10.4000/ifha. 8043

ISSN : 2198-8943

\section{Éditeur}

IFRA - Institut franco-allemand (sciences historiques et sociales)

\section{Édition imprimée}

Date de publication : 31 décembre 2014

ISSN : 2190-0078

\section{Référence électronique}

Věra Vejrychová, "Le concept de la nation chez Jean Froissart », Revue de l'IFHA [En ligne], 6 | 2014, mis en ligne le 31 décembre 2014, consulté le 02 mai 2019. URL : http://journals.openedition.org/ ifha/8043 ; DOI : 10.4000/ifha.8043

Ce document a été généré automatiquement le 2 mai 2019.

(CIFHA 


\title{
Le concept de la nation chez Jean Froissart
}

\author{
Věra Vejrychová
}

1 Malgré des opinions toujours très variées sur la nature du sentiment national avant l'époque moderne, il est impossible de nier la conscience tout à fait nette des médiévaux de la diversité du monde, conçu suite à l'héritage antique comme une pluralité de pays et de leurs habitants ${ }^{1}$. En analysant les Chroniques de Jean Froissart de ce point de vue, nous souhaitons non seulement de suivre sa façon dire le multiple et de le classer en unités organisationnelles appelées nations, mais aussi nous demander si, pour lui, cette catégorie descriptive devient une catégorie fonctionnelle, une catégorie où la nation apparaît comme une unité cohérente agissant dans le sens même de ce qui la définit, ou bien ce qui la distingue par rapport au reste du monde. Pour répondre à cette question fondamentale, nous nous attacherons d'abord au problème de l'appelation. L'analyse lexicologique permettra de comprendre de quelle façon sont abordées les différentes identités. Cela nous amènera ensuite à une réflexion sur les éléments constitutifs de ces liens de solidarité. Et enfin, il n'est possible de comprendre l'enjeu du concept de la nation et de son rôle dans le récit historique des Chroniques, qu'à travers les propres sentiments d'appartenance de l'auteur et à travers d'autres influences qui structurent la vision de la réalité qui est la sienne.

\section{Nommer la nation}

2 Les Chroniques de Froissart sont remplies de personnages de différentes origines et de communautés de gens, les unes et les autres perçues comme des entités aux traits caractéristiques propres. Nommer un tel ensemble de population pose le problème du glissement de sens dans les textes vernaculaires par rapport à la signification originelle du terme en $\operatorname{latin}^{2}$. Ainsi, le mot « gens " peut se rapporter chez Froissart à la population d'un pays, mais il a toujours et d'abord son sens le plus général. Le mot "communauté » n'apparait que dans le sens social du commun. Quant au mot " nation », son utilisation est 
très fréquente chez Froissart. Dans une étude datant de 1947, Franz Müller interprète cette récurrence inhabituelle par l'intérêt particulier que le chroniqueur portait à la question de la nation ${ }^{3}$. Mais, pour pouvoir véritablement discerner sa spécificité éventuelle, il est nécessaire de confronter les significations assez variées que le mot "nation" peut revêtir chez cet auteur, avec les usages dans d'autres sources historiographiques de l'époque. Les chroniques vernaculaires témoignent pour leur part d'une appropriation extrêmement timide du mot. Seulement quelques occurrences peuvent être relevées, et elles se rapportent toutes à l'indication de l'origine multiple dans des armées composites ${ }^{4}$. C'est donc le sens premier, tel qu'il est défini par Isidore de Séville, que l'on trouve attesté en français : "nation» exprime la relation étroite de l'individu avec sa terre d'origine ${ }^{5}$. Cet emploi est plus répandu dans les textes latins de l'époque ${ }^{6}$. Le mot "natio" y apparaît également pour exprimer la multitude et la diversité. Il peut se rapporter aussi bien à une population qu'à un pays ${ }^{7}$. On ne trouve toutefois jamais l'expression « natio Franciae » alors que « gens Franciae » est attesté ${ }^{8}$.

Chez Froissart dominent exactement les mêmes usages et significations. La fréquence beaucoup plus élevée de leur emploi tient surtout à la matière de son récit. Le mot "nation" se rapporte chez lui tout au long du texte à des identifications urbaines, régionales et étatiques. Ces significations correspondent évidemment aux différents types de sentiments de solidarité, qui ne s'excluent pas mutuellement, mais se superposent et s'entrecroisent ${ }^{9}$. Les syntagmes "d'estragnes nations", "autres nations", "de quelconques nations qu'ils fuissent", dans la plupart des occurrences, signifient l'origine, le pays d'origine ou le pays dans le sens général ${ }^{10}$. C'est aussi souvent le cas dans les expressions « nation de France », « nation d'Angleterre », « nation d'Espagne » qui - nous y insistons ne furent pas trouvées ailleurs, ni en français, ni en latin ${ }^{11}$. Plus rares sont les emplois où ces expressions indiquent les populations. Cet usage n'est guère attesté dans le livre I des Chroniques, sauf dans la rédaction tardive dite de Rome ${ }^{12}$. À partir du livre II, il apparaît avec une fréquence régulière mais faible ${ }^{13}$. Il est donc sûr que cet emploi est chez Froissart postérieur à 1386. Il ne se trouve pas dans les parties copiées sur la Chronique de Jean le Bel, elle-même composée au cours des années cinquante pour sa plus grande partie $^{14}$, ni dans ses propres développements du premier livre dans les deux premières rédactions. Sans prétendre à l'exhaustivité de cette analyse lexicologique, il semble possible de conclure d'une part à une utilisation plutôt très timide de ce terme dans le sens national pour cette période, et d'autre part à son évolution au cœur même des Chroniques, ce qui peut refléter une maturation du concept de la nation chez Jean Froissart, comme nous essaierons de le montrer dans les développements ultérieurs.

\section{Définir la nation}

Le problème des nations au Moyen Âge est pensé dans l'historiographie moderne notamment par rapport à la (pré)existence de l'État, au rôle de la société politique, et aux mythes des origines avec leur fonction génératrice d'un imaginaire de la nation. Les critères imposés par le nationalisme moderne, le partage culturel et l'unité linguistique, dont la littérature vernaculaire serait l'un des vecteurs principaux, sont aussi au cœur du questionnement. Froissart traite de tous ces éléments. Il s'agit donc de voir à quel point ceux-ci sont envisagés comme constitutifs d'une nation.

5 Le sentiment national comme le résultat de la jonction des solidarités verticales et horizontales se manifeste de façon évidente dans les descriptions de la déloyauté 
politique. Les personnes qui font preuve d'un tel comportement sont à plusieurs reprises qualifiées péjorativement de "mauvais François", de "mescheans gens »" Leur infidélité est articulée à travers l'idée de la trahison de leur pays d'origine, qui n'est pas alors défini géographiquement comme une région, mais politiquement comme un État. Ainsi le lieu de naissance est-il inséparablement attaché à l'appartenance à un corps politique. Et cette appartenance est comprise en termes de sentiment. Froissart joue sur le registre des émotions entre le seigneur et ses sujets surtout dans la situation postérieure à la conclusion du traité de Brétigny/Calais, lorsque certaines régions devaient passer sous la souveraineté du roi d'Angleterre. Les élites des villes et la noblesse se montrent alors porteurs et garants de la fidélité dynastique, qui constitue au Moyen Âge l'un des fondements du sentiment national. De manière générale, rien ne permet de considérer que la jonction de la solidarité d'ordre politique à celle définie par l'origine ne soit seulement naturelle. Elle suppose au contraire l'existence d'un cadre étatique, comme l'affirmait Bernard Guenée, dans lequel peut se mouler un amor patriae. En revanche, les situations dans lesquelles cette condition n'est pas remplie peuvent donner naissance à de véritables concurrences entre ces deux sentiments.

6 Froissart thématise cette question notamment pour la grande Guyenne, où s'imposèrent dans les années 1360 de nouvelles exigences de fidélité. L'entrelacement et la hiérarchie des solidarités diverses sont condensés dans la réponse des barons d'Aquitaine au prince de Galles sur le plan de la campagne en Castille. Les seigneurs voudraient en tirer le bénéfice parce qu'elle ne se ferait pas pour « les besongnes de nostre chier signeur vostre père ou pour les vostres ou pour vostre honneur ou de nostre pays... $»^{16}$. La notion de l'honneur de " nostre pays »- pays qui n'est pas identique à celui de leur roi - est ainsi juxtaposée à d'autres loyautés. Cette dichotomie devient très tôt conflictuelle. Non qu'il s'agisse d'une lutte nationale parce que les tensions sont de nature politique ou économique, mais la question de la nationalité devient un vecteur et peut-être aussi un catalyseur du conflit. L'entourage anglais du prince se présente en effet comme étranger par rapport aux populations locales, ce qui se traduit par une monopolisation absolue des offices ${ }^{17}$. La coexistence de deux communautés d'origine différente est signalée également par le bilinguisme. De la même façon que la distribution des offices est fermée à tous les nonAnglais, la communication l'est également lorsque le prince de Galles souhaite ne pas se faire comprendre en dehors de son entourage anglais ${ }^{18}$. Ainsi la langue est-elle l'élément essentiel de la culture partagée. Elle joue un rôle d'unification face à une communauté linguistique étrangère et constitue le moyen de faire valoir sa propre nationalité et le pouvoir politique qui en découle. L'attention que Froissart porte à la question de la langue s'aiguise vers la fin des Chroniques, et c'est surtout l'anglais dont il se préoccuppe ${ }^{19}$. Il le perçoit en relation dichotomique avec le français, et le progrès de l'un devient forcément dévastateur pour l'autre. Il ne se pose pas véritablement la question de savoir si c'est la langue qui crée la nation ou la nation qui impose l'unification linguistique, mais pour lui, ces deux processus sont connexes.

7 Contrairement à son intérêt pour le rôle de la langue, il passe entièrement sous silence les mythes d'origine conçus comme source d'identité collective. Les Chroniques contiennent par ailleurs très peu de développements au sein desquels l'histoire est instrumentalisée pour démontrer une appartenance ${ }^{20}$. L'un des rares exemples est contenu dans l'argumentation des Anglais affirmant que l'Écosse doit relever du roi anglais parce que les deux royaumes furent jadis gouvernés par un seul roi ${ }^{21}$. L'attachement de l'Écosse à l'Angleterre, quoique justifié aux yeux des Anglais par la même langue et par l'espace 
unifié du droit, ne se ferait pas sur le plan national. Il se ferait uniquement sur le plan politique. Froissart n'a donc aucune difficulté à penser deux nations à l'intérieur d'un État, comme dans le cas de la Guyenne anglaise. Il n'en reste pas moins qu'une telle coexistence peut facilement devenir source de conflits.

\section{Perception de différentes nations}

$8 \quad$ Les commentaires sur des nationalités différentes ne sont pas proposés comme de simples perceptions et conclusions de l'auteur. Souvent filtrés par le regard des représentants d'autres pays, ils entrent dans le jeu de miroirs, dont l'effet participe évidemment de la construction narrative. C'est donc l'enjeu du discours, la position de l'énonciateur dans le récit, mais aussi l'identité de la source d'informations et finalement l'identité propre du chroniqueur qui structurent de manière complexe ce que nous lisons à propos de différentes nations.

Jean Froissart se définit par deux sentiments d'appartenance fondamentaux. Le premier tient à son identité formée et véhiculée par sa langue maternelle. Le français est pour lui le moyen d'exister dans différents pays sans s'y sentir étranger, et il possède donc forcément une fonction d'intégration ${ }^{22}$. Le deuxième élément propre à constituer un sentiment d'appartenance réside dans son pays d'origine. À partir du moment où le chroniqueur met en scène sa personne, ses entretiens, ses voyages et ses souvenirs, les références à «sa nation » deviennent très fréquentes, presque exclusivement en liaison avec le travail de l'écriture. Les retours dans son pays d'origine lui permettent en effet de rédiger le texte des Chroniques à partir de ses notes. Certes, il n'y revient pas porté par un sentiment de mal de pays, mais tout simplement parce que c'est là où se trouve sa prébende, sur laquelle repose sa forge littéraire. Mais l'aspect pratique se conjugue avec une affinité. Il y a, chez Froissart, un concept implicite d'amor patriae, rapporté avant tout à lui-même. Mais s'il s'identifie avec le Hainaut, il ne le fait jamais avec les Hennuyers en tant qu'un ensemble national.

10 Au cœur de l'identité chrétienne, culturelle et linguistique, qui forme sa perception du propre et de l'étranger, les nations apparaissent comme la figure de l'altérité. La façon de les envisager tient chez Froissart dans une combinaison de la reproduction des hétérostéréotypes et de l'équation selon laquelle le plus éloigné, le plus exotique, mérite la description la plus détaillée. Ce sont les nations placées dans l'orbite extrême du monde chrétien auxquelles il consacre des récits quasi ethnographiques. Il le fait pourtant en une très grande dépendance envers ses sources d'informations. Par conséquent, les Chroniques contiennent certains récits parallèles des mêmes réalités, mais qui sont parfois incohérents les uns avec les autres ou avec sa propre expérience du voyageur (visite d'Écosse en 1365). Sur l'autre pôle du spectre de l'étrangeté ne sont pas placés exclusivement les Hennuyers, en tant que siens compatriotes, mais tous les peuples de la sphère francophone. Les frontières linguistico-culturelles empiètent de manière décisive sur les frontières géo-politiques. Les principautés et seigneuries à la frontière impériale avec la France (Brabant, Hainaut, Cambrésis etc.) sont, dans l'esprit de Froissart, vaguement attachées à ce grand ensemble étatique qu'est l'Empire, mais se distinguent profondément de la majorité absolue (germanophone et autres), de ses habitants, perçus comme autres, différents, comme une masse sans différenciation verticale, les nobles comme le peuple étant porteurs des mêmes traits caractéristiques peu appréciés ${ }^{23}$. En revanche, tout ce qui relève de l'identité propre du chroniqueur n'est 
pas soumis à un jugement des communautés nationales. Il omet entièrement une caractéristique des Hennuyers, et de manière analogique, nous constatons l'absence d'une démarche d'évaluation à l'égard des Français.

11 La seule nation à subir une évolution en fonction de son déplacement sur la ligne du connu vers l'étranger, est celle des Anglais. L'idée de leur prouesse et de leur vaillance exceptionnelles traverse le texte entier des Chroniques, mais ces qualités sont petit à petit investies d'aspects négatifs, tels que le caractère belliqueux, l'orgueil ${ }^{24}$... Le chroniqueur multiplie d'abord les témoignages de ceux qui connaissent la véritable nature des Anglais, pour s'y associer enfin lui-même dans la dernière rédaction du premier livre ${ }^{25}$. La visite de l'Angleterre en 1395 confronta un Froissart devenu nostalgique à une nouvelle réalité, et cette vision de la nation définie d'abord par une altérité linguistique, prend définitivement corps sous le coup de la déposition de Richard II en 1399. Si Froissart signale au début du manuscrit de Rome une grande différence entre les nobles et le peuple anglais ${ }^{26}$, en réalité, il dépeint souvent les seigneurs d'Angleterre exactement dans les mêmes couleurs mornes. Ce n'est donc pas la «bourgeoisie agressive ", comme l'affirmait Franz Müller, qui serait seule porteuse du caractère national. Au contraire, l'effort de nuance sociale, que nous avons pu constater et ériger en signe de la perception interne du chroniqueur, s'efface de façon importante. Ainsi la description tardive des Anglais représente-t-elle le regard le plus complexe sur un comportement conçu véritablement comme national, mû par une nature commune à tout le pays, et influençant sur le déroulement de l'histoire, parce qu'agissant au profit de l'ensemble, ou de ce qui est présenté comme tel.

La matière géographiquement assez dispersée des Chroniques amène Jean Froissart à envisager la multiplicité politique, ethnique, linguistique et culturelle, ce qui explique aussi la fréquence très grande du terme «nation ». Il le fait, bien évidemment, en fonction de sa propre identité. Le jeune chroniqueur, éprouvant d'abord surtout un sentiment d'appartenance envers son pays d'origine et, à la limite, une solidarité avec ses ressortissants, ne pouvait à partir de ses propres structures mentales initiales aller audelà de la dimension transnationale de son identité francophone. Sa représentation des nations est biaisée par le poids des stéréotypes qui reviennent surtout dans les jugements des peuples qui ne sont pas perçus comme très lointains, mais comme hiérarchiquement inférieurs. Les nations plus exotiques sont décrites de manière plus complexe. Mais il reste que pour ce genre d'informations, le chroniqueur est toujours entièrement dépendant de ses sources. C'est aussi la raison pour laquelle sa représentation des nations se construit surtout par rapport au comportement dans la guerre. Au cours des Chroniques , un développement de l'idée de la nation se manifeste de façon distincte. Ce n'est pourtant pas tant la découverte d'autres pays, régions et peuples à travers l'expérience du voyage, qui formait sa perception de l'étranger et contribua à une maturation du concept de la nation. C'est bien davantage la rencontre avec d'autres façons d'envisager ces réalités, avec d'autres discours sur l'identité, qui lui permet de voir derrière les royaumes et les dynasties des peuples, avec leurs propres sentiments d'appartenance et la manière de se définir par rapport aux autres communautés, souvent les plus proches. Et ensuite, de façon décisive, ce fut la confrontation avec la nouvelle réalité anglaise, contrastant avec son expérience de jeunesse qui transforma définitivement en certitude sa prise de conscience progressive de l'importance de l'unité linguistique d'une communauté, au sein de laquelle la fidélité politique, résultat naturel d'un attachement au pays d'origine, fusionne de façon nouvelle avec d'autres éléments participant d'une même identité. Ainsi 
émerge le concept de la nation, unie et politiquement active au service de son bien commun. Mais ceci était possible uniquement parce que, pour Froissart, l'Angleterre devint autre.

\section{NOTES}

1. Cette étude a été réalisée dans le cadre du projet « Les codes culturels et leurs transformations à l'époque hussite » P405/12/G148 soutenu par l'Agence tchèque de la recherche scientifique (GAČR).

2. Bernard Guenée, «État et nation en France au Moyen Âge ", Revue historique, n 237, 1967, p. 17-30.

3. Franz Walter Müller, «Zur Geschichte des Wortes und Begriffes 'nation' im französischen Schrifttum des Mittelalters bis zur Mitte des 15. Jahrhunderts", Romanische Forschungen, $\mathrm{n}$ - 58/59, 1947, p. 247-321, surtout p. 301-310.

4. Roland Delachenal (éd.), Chronique du règne de Jean II et Charles V, Paris : SHF, 1916, t. 2, p. 10, 15, 27.

5. Bernard Guenée, «État et nation en France au Moyen Âge », art. cit.

6. Hercule Géraud (éd.), Chronique latine de Guillaume de Nangis avec les continuations, Paris: SHF, 1843 , t. 2, p. 15, 24, 50, 122 ; Henri Moranvillé (éd.), Chronographia regum francorum, Paris : SHF, 1893, t. 2, p. 71, 374 ; Jean Lemoine (éd.), Chronique de Richard Lescot, Paris : SHF, 1896, p. 186, 187, 189.

7. Hercule Géraud (éd.), Chronique latine de Guillaume de Nangis, op. cit., t. 2, p. 245, 298, 316 ; Henri Moranvillé (éd.), Chronographia, op. cit., t. 2, p. 286 ; Jean Lemoine (éd.), Chronique de Richard Lescot, p. 149.

8. Hercule Géraud (éd.), Chronique latine de Guillaume de Nangis, op. cit., p. 210.

9. Reiner Babel/Jean-Marie Moeglin (dir.), Identité régionale, Conscience nationale en France et en Allemagne du Moyen Âge à l'époque moderne, Sigmaringen : J. Thorbecke, 1997, p. 9.

10. Siméon Luce/Gaston Raynaud/ Léon Mirot (éd.), Chroniques de J. Froissart, Paris: SHF, $1868-1975$, t. 6 , p. 60,141 ; t. 7, p. 181 ; t. 9 , p. 156 ; t. 10 , p. 101,188 ; t. 11 , p. 166 ; t. 12 , p. 155 ; t. 14, p. 5, 135-136; George Diller (éd.), Chroniques. Livre I. Le manuscrit d'Amiens, Genève : Droz, 1991-1998, t. 4, p. 344 ; Kervyn de Lettenhove (éd.), CEuvres de Froissart, Bruxelles: V. Devaux, $1867-1877$, t. 14 , p. 2, p. 155 ; t. 16, p. 55, p. 148, p. 189, p. 190.

11. Bernard Guenée parle de l'usage fréquent de l'expression « nation de France » en se référant à l'analyse de Gustave Dupont-Ferrier, «Sur l'emploi du mot 'province' notamment dans le langage administratif de l'ancienne France», Revue historique, $\mathrm{n}^{\circ} 161,1929$, p. 278-303. Ses exemples sont pourtant plus tardifs, datant du XVe siècle.

12. George Diller (éd.), Chroniques. Dernière rédaction du premier livre, Genève/Paris : Droz/Minard, 1972, p. 221, 887.

13. Siméon Luce/Gaston Raynaud/Léon Mirot (éd.), Chroniques de J. Froissart, t. 10, p. 172 ; t. 11, p. 86 ; t. 13, p. 216 (cf. CEuvres de Froissart, t. 12, p. 146 et le ms. de Besançon 865, fo331, accessible sur http://www.hrionline.ac.uk/onlinefroissart) ; t. 15, p. 13 ; Kervyn de Lettenhove (éd), Cuvres de Froissart, op. cit., t. 15, p. 117, 124, 218, 330.

14. Cf. Jules Viard/Eugène Déprez (éd.), l'introduction dans la Chronique de Jean Le Bel, Paris : SHF, 1904. 
15. Siméon Luce/Gaston Raynaud/Léon Mirot (éd.), Chroniques de J. Froissart, op. cit., t. 6, p. 60 ; t. 8 , p. 112 ; Kervyn de Lettenhove (éd), Euvres de Froissart, op. cit., t. 14, p. 287-290.

16. Siméon Luce/ Gaston Raynaud/Léon Mirot (éd.), Chroniques de J. Froissart, op. cit., t. 6, p. 207.

17. Ibid., t. 7, p. 84-85.

18. Ibid., t. 6, p. 218, 232.

19. Georges Diller (éd.), Chroniques. Dernière rédaction du premier livre, op. cit. , p. 211, 232 ; Kervyn de Lettenhove (éd), CEuvres de Froissart, op. cit., t. 15, p. 114-115.

20. Siméon Luce/Gaston Raynaud/Léon Mirot (éd.), Chroniques de J. Froissart, op. cit., t. 6, p. 57.

21. Georges Diller (éd.), Chroniques. Livre I. Le manuscrit d'Amiens, t. 1, p. 122.

22. Sans qu'il soit possible d'affirmer le monolinguisme de Froissart, il n'est pas non plus possible d'affirmer le contraire. À notre connaissance, les Chroniques ne fournissent aucun élément permettant de dire que Froissart pouvait interroger ses informateurs dans d'autres langues comme l'affirme Zrinka Stahuljak, «Jean Froissart's Chroniques: Translatio and the Impossible Apprenticeship of Neutrality ", in : Renate Blumenfeld-Kosinski/Luise von Flotow/Daniel Russell (dir.), The Politics of Translation in the Middle Ages and Renaissance, Ottawa : University of Ottawa Press, 2001, p. 121-142. Voir aussi Georges Diller, «Pour la cause de ce que j'estoit françois. Langue(s) et loyauté(s) dans les Chroniques de Froissart », Moyen Âge, n 104, 1998, p. 461-472.

23. Signalons seulement le déplacement $d u$ « centre » d'une telle vision en fonctions de la source. Tel est le cas pour Bernat Desclot, un chroniqueur catalan, qui considère à propos des vêpres siciliennes que les soldats français au service des Angevins n'étaient pas civilisés, possédaient de rudes manières, déclenchaient que des violences sexuelles. Cf. Jaume Aurell, Authoring the Past, Chicago ; London : Chicago University Press, 2012, p. 68.

24. Siméon Luce/Gaston Raynaud/Léon Mirot (éd.), Chroniques de J. Froissart, op. cit., t. 8, p. 161-162.

25. George Diller (éd.), Chroniques. Dernière rédaction du premier livre, op. cit., p. 220-221.

26. Ibid., p. 42-43.

\section{AUTEUR}

\section{VĚRA VEJRYCHOVÁ}

(Université Paris-Sorbonne/Université Charles de Prague) 